\title{
Analytic theory of hexagonal monolayer interacting with hexagonal substrate
}

\author{
Alexandre Tkatchenko* \\ Departamento de Química, División de Ciencias Básicas e Ingeniería, Universidad Autónoma Metropolitana-Iztapalapa, \\ Av. San Rafael Atlixco 186 Col. Vicentina, AP. 55-534, México D.F. 09340, Mexico \\ (Received 14 March 2006; revised manuscript received 29 May 2006; published 26 July 2006)
}

\begin{abstract}
Analytic theory of the infinite rigid hexagonal monolayer interacting with hexagonal substrate is developed. The interaction potential is described by a Fourier series with up to six shells of the reciprocal cell vectors. It is rigorously shown that energy of minimum structures is directly related to their symmetry, assuming a decaying behavior of Fourier coefficients. Preliminary comparison with real systems of halogens and alkalis adsorbed on metal surfaces is encouraging and indicates that the adsorbate-substrate interaction is one of the main driving forces in the monolayer formation. The need of detailed $a b$ initio calculations to obtain the Fourier coefficients for different complex adsorption systems is emphasized. Our results indicate the importance of accurate description of the adsorbate-substrate interactions for quantitative theory of monolayer epitaxy on well-ordered surfaces.
\end{abstract}

DOI: 10.1103/PhysRevB.74.035428

PACS number(s): 68.35.Md, 71.15.Ap, 31.15.Ew, 31.30.-i

\section{INTRODUCTION}

Monolayers adsorbed on well-ordered substrates show a wide variety of interesting behavior, such as rotational and translation epitaxy, and have been of interest in experimental and theoretical research for a long time. ${ }^{1}$ The detailed description of possible structures of adsorbed monolayers and phase transitions between them has received considerable attention in the literature, with square monolayers adsorbed on square or rectangular substrates being one of the most studied systems. ${ }^{2-4}$ The matrix description of the structure of adsorbed monolayers respective to the substrate allows one to define several monolayer classes., ${ }^{5,6}$ Many different adsorption systems possess hexagonal monolayer structures on substrates with hexagonal symmetry: rare gases on metal surfaces and graphite, halogens on metal surfaces, alkalis on metal surfaces, among others. Usually these systems show interesting behavior of rotational epitaxy and many models have been proposed up to date to describe them. The Novaco-McTague model is the most well-known and accepted one. ${ }^{7,8}$ It describes the monolayer in terms of the elastic constants and shows that the ground state of an adsorbed monolayer could be at a nonsymmetry angle respective to the substrate. The interaction of the adsorbate layer with the substrate is assumed to be very weak and the rotational epitaxy is mainly driven by the interactions between the adsorbate atoms. A different model was proposed by Doering, ${ }^{9}$ who used symmetry considerations to explain the rotational behavior of an adsorbed monolayer. Grey and Bohr ${ }^{10,11}$ used a one Fourier component model for the adsorbed atom interaction with the surface and proposed the finite-size effect due to surface terraces as an important contribution to rotational epitaxy. Furthermore, Shiba ${ }^{12,13}$ proposed a theory based on domain walls to explain the behavior of rare gases adsorbed on the metal surface. These models predict qualitatively different behavior for the rotation angle of the adsorbed monolayer. Despite of a large number of published models, many experimentally well-known systems cannot be described in detail by any of them. One clear example is the alkalis on the $\mathrm{Ag}(111)$ surface. ${ }^{14}$ The rotation angle behavior for hexago- nal monolayers of $\mathrm{K}, \mathrm{Rb}$, and $\mathrm{Cs}$ adsorbed on $\mathrm{Ag}(111)$ are markedly different than those predicted by any of the abovementioned models. Another example is rare-gas monolayers on the $\operatorname{Ag}(111)$ surface, ${ }^{15}$ where it has been proposed that adsorbate-substrate interaction plays an important role. The quantitative behavior of halogen monolayers on $\operatorname{Pt}(111)$, $\mathrm{Au}(111)$, and $\operatorname{Ag}(111)$ surfaces also remains unexplained from the theoretical point of view. ${ }^{16-19}$ One aspect that seems to be similar in all of the previous models of rotational epitaxy of adsorbed monolayers is that the interaction of the adsorbed atom with the substrate is assumed to be weak or described by a single term of the Fourier expansion. Although such approximation has been shown to be valid for simple pairwise interactions between the adsorbate and substrate atoms, ${ }^{20}$ and confirmed for systems such as rare-gases on graphite and $\mathrm{Xe}-\mathrm{Pt}(111),{ }^{21-23}$ in the case of more complex interactions, accurate description of the atom-surface potential requires higher-order Fourier terms. ${ }^{24}$

The Fourier expansion technique allows us to describe the atom-surface interaction potential with prescribed accuracy. ${ }^{20,24}$ While it is not clear how to obtain analytic expressions for the adsorbate-adsorbate interactions from $a b$ initio calculations, the Fourier coefficients could be obtained in principle. The Fourier expansion of the adsorbate-substrate interaction potential has been applied in many different studies, having the most success for physisorption systems. ${ }^{25}$ The Fourier expansion is a completely general technique in the sense that it can be used to describe any function with the symmetry of the underlying surface.

In this paper, analytic theory of the infinite rigid hexagonal monolayer adsorbed on a well-ordered hexagonal surface is developed. The interaction between adsorbate atoms and the substrate is represented by a Fourier expansion with a successively larger number of terms, up to six shells of reciprocal vectors. It is shown that each Fourier term contributes to the stability of a finite number of simply commensurate or high-order commensurate (HOC) structures. The qualitative results do not depend on the values of the Fourier coefficients. It is clearly shown that the symmetry of the adsorbed monolayer is directly related to its energy, assum- 
ing a simple decaying behavior of the coefficients. We extend the models of Grey-Bohr and Doering, by showing that many different paths between HOC structures could exist in the configuration space of hexagonal monolayers. Preliminary comparison is made with real systems of halogens and alkalis on metal surfaces and several interesting trends are identified. However, quantitative description of any real system requires the knowledge of the Fourier coefficients. Our analysis indicates that an accurate description of adsorbatesubstrate interactions is required for quantitative theory of monolayer epitaxy on well-ordered surfaces.

The organization of this paper is as follows: In Sec. II the origin of hexagonal monolayers and the rigid approximation is discussed, Sec. III presents the development of the analytic theory, in Sec. IV a preliminary comparison with real experimental systems is done, Sec. V presents a discussion of the obtained results. Finally, conclusions are made in Sec. VI.

\section{HEXAGONAL MONOLAYERS AND RIGID APPROXIMATION}

The hexagonal monolayer structures on well-ordered surfaces are formed as result of minimization of the repulsive part of the interaction potential between the adsorbate atoms. In the case of purely repulsive adsorbate-adsorbate interaction, the hexagonal monolayers are presumably the most stable ones and simple commensurate structures would be exclusively seen in experiments due to the effect of adsorbate-substrate interactions. It is assumed in this study that the lateral interaction in the monolayer is strong enough to allow formation of rigid hexagonal arrangements, and further accommodation is driven by the adsorbate-substrate interaction exclusively. On the other hand, complex adsorbateadsorbate interactions could induce the rich behavior observed in many adsorption systems, for example, the $C$ $\rightarrow S I \rightarrow H I \rightarrow H I R$ structure sequence for $\mathrm{Xe}-\mathrm{Pt}(111) .{ }^{26}$ The large number of models proposed to date to explain the rotational epitaxy of adsorbed monolayers allow us to conclude that many different phenomena are important, namely the adsorbate-adsorbate interactions, finite-size effects of the surface terraces, symmetry considerations, among others. The one ingredient that seems to be overlooked by many existing models is the detailed description of the adsorbate-substrate interaction, i.e., the contribution of the high-order Fourier terms, which is analyzed in this paper.

In order to verify the validity of the rigid approximation for the monolayer description in the case of real monolayersurface systems, we proceed to analyze the magnitudes of the adsorbate-substrate and the adsorbate-adsorbate interactions in the case of $\mathrm{I}-\mathrm{Pt}(111),{ }^{27,28} \mathrm{Xe}-\mathrm{Pt}(111),{ }^{29,30}$ and $\mathrm{Xe}-$ $\operatorname{Pd}(111)$ (Ref. 30) systems, for which DFT calculations for top, fcc, and bridge sites are available. That allows us to calculate the first three coefficients of the Fourier expansion. Unfortunately, the description of adsorption energies for different low-symmetry sites on the substrate, which would allow detailed analysis of the Fourier expansion, is available for very few adsorption systems. The I-Pt(111) system is chosen as an example of halogen adsorption on metal surfaces, which will be compared with results of the analytic theory later. $\mathrm{Xe}-\mathrm{Pt}(111)$ and $\mathrm{Xe}-\mathrm{Pd}(111)$ are chosen as examples of rare-gas adsorption on metal surfaces, for which analytic expressions for adsorbate-adsorbate interactions are well known. ${ }^{31,32}$ In order to see if the usual one-term Fourier expansion with $V_{G_{0}}$ coefficient is a good approximation to the adsorbate-substrate interaction in these systems, the $\left(E_{\text {atop }}-E_{\text {bridge }}\right) /\left(E_{\text {atop }}-E_{f c c}\right)$ ratio is used. This ratio for a one-term expansion should be around 0.89 . For I-Pt(111), ${ }^{27}$ it is 0.78 , for $\mathrm{Xe}-\mathrm{Pt}(111),{ }^{29}$ it is 0.92 , and for $\mathrm{Xe}-\mathrm{Pd}(111),{ }^{30}$ it is 0.76 . It can be seen that for the $\mathrm{Xe}-\mathrm{Pt}(111)$ case, the one-term Fourier expansion is a very good approximation, while for I-Pt(111) and Xe-Pd(111) systems, the same cannot be concluded. Furthermore, in the I-Pt(111) case, $V_{\sqrt{3} G_{0}}$ $=-0.24 V_{G_{0}}$, while for $\mathrm{Xe}-\operatorname{Pt}(111) V_{\sqrt{3} G_{0}}=0.05 V_{G_{0}}$ and for $\mathrm{Xe}-\operatorname{Pd}(111) V_{\sqrt{3} G_{0}}=-0.08 V_{G_{0}}$. From this simple analysis, it seems that the Fourier expansion converges faster for physisorption systems. However, a careful systematic study is required to confirm this conjecture.

From experimental point of view, the commensurate $(\sqrt{3}$ $\times \sqrt{3}) R 30^{\circ}$ and $(\sqrt{7} \times \sqrt{7}) R 19.11^{\circ}$ structures have been found for I-Pt(111) (Ref. 16) and Xe-Pd(111) (Refs. 33-35) systems, while only $(\sqrt{3} \times \sqrt{3}) R 30^{\circ}$ has been found for Xe$\mathrm{Pt}(111) .{ }^{36}$ Additionally, I-Pt(111) shows the commensurate $(3 \times 3)$ structure $^{16}$ and $\mathrm{Xe}-\mathrm{Pd}(111)$ shows the high-order commensurate (HOC) $(\sqrt{19} \times \sqrt{19}) R 23.4^{\circ}$ structure. $^{35} \mathrm{Al}-$ though the appearance of HOC structures for Xe-Pd(111) is thought to happen due to residual surface contamination with impurities, the HOC structures have been found in several studies and the contamination should mainly block the surface steps and can influence the effective lateral interaction as it has been conjectured for the $\mathrm{Xe}-\mathrm{Ag}(111)$ monolayer. ${ }^{15}$

In the next section it will be shown that the first Fourier term (the one that generally contributes the most) vanishes for a rigid hexagonal structure with more than one atom per unit cell. Therefore in the vicinity of the rigid hexagonal arrangement it should have a very small contribution. On the contrary, the first Fourier term is fully conserved for the $(\sqrt{3} \times \sqrt{3}) R 30^{\circ}$ structure and the second Fourier term is fully conserved for a rigid hexagonal $(\sqrt{7} \times \sqrt{7}) R 19.11^{\circ}$ structure with three atoms per unit cell. Considering that these structures have been observed in experiments for I-Pt(111) and Xe-Pd(111), we could use the data about magnitudes of adsorbate-substrate and adsorbate-adsorbate interactions to estimate the necessary conditions for the theory in this paper to be valid. The HFD-B2 model ${ }^{32}$ augmented by McLachlan dispersion ${ }^{37}$ has been frequently used for the description of lateral interactions of the rare-gas monolayers on metal surfaces. ${ }^{31}$ The magnitude of lateral interactions for the Xe$\operatorname{Pd}(111)$ system should be similar to Xe-Pt(111). Therefore we will use the values reported in Ref. 31 for the Xe-Xe interaction. The minimum of the HFD-B2 potential with McLachlan dispersion for $\mathrm{Xe}-\mathrm{Xe}$ is at $1.59 l$, where $l$ is the substrate lattice constant. The interatomic distance for $(\sqrt{3}$ $\times \sqrt{3}) R 30^{\circ}$ structure is $\sqrt{3} l$ with potential gradient of $21 \mathrm{meV}^{-1}$ at this point (attractive part of the interaction) while for $(\sqrt{7} \times \sqrt{7}) R 19.11^{\circ}$ the distance is $1.53 l$ with gradient of $-32 \mathrm{meVl}^{-1}$ and lies on the repulsive part of the interaction curve. The total lateral interaction energy for rigid 
$(\sqrt{7} \times \sqrt{7}) R 19.11^{\circ}$ is $-58.7 \mathrm{meV}$, while the second Fourier coefficient in $\mathrm{Xe}-\mathrm{Pd}(111)$ is $0.83 \mathrm{meV}$. It is feasible to think that the effective lateral interaction energy does not change its magnitude due to the blocking of the surface steps. In the case of I-Pt(111) system, the gradient for $(\sqrt{7} \times \sqrt{7}) R 19.11^{\circ}$ structure is around $-301 \mathrm{meV} l^{-1}$ and the total interaction energy is of $205 \mathrm{meV},{ }^{28}$ while the second Fourier coefficient is $-23.22 \mathrm{meV}$. It can be seen that the $\left|V_{\sqrt{3} G_{0}} / \Delta E_{\text {lateral }}\right|$ ratio for $(\sqrt{7} \times \sqrt{7}) R 19.11^{\circ}$ varies from 0.03 for $\mathrm{Xe}-\mathrm{Pd}(111)$ to 0.08 for I-Pt(111). Assuming that the experimentally found structures are rigid, the theory presented in this work should be accurate for such magnitudes of interaction.

It should be kept in mind that the performed analysis is merely of qualitative nature, however, it should be possible to assign quantitative limits for the validity of rigid approximation after a careful study relaxing the rigidity constraint in different unit cells and for different interaction models.

\section{ANALYTIC THEORY OF HEXAGONAL MONOLAYER INTERACTING WITH HEXAGONAL SUBSTRATE}

The interaction of an adsorbate atom with the substrate surface is expanded in Fourier series, ${ }^{20,25}$

$$
V(\mathbf{r})=V_{0}+\sum_{G} V_{G} \exp (-1 \mathbf{G r}),
$$

where $\mathbf{r}$ is the real space vector, $\mathbf{G}$ is the reciprocal space vector, and $V_{G}$ are the Fourier coefficients. The $V_{G}$ coefficients usually depend on the vertical $z$ position of the atom on top of the surface, however, the qualitative results of the present study do not depend on the form of the $V_{G}$ coefficients. Moreover, a quasi-2D model of the monolayer is assumed and the $V_{G}$ values are taken at the equilibrium $z$ distance, when appropriate. The difference in adsorbatesubstrate interactions between adsorption systems is "encoded" in the Fourier coefficients. In the case of simple pairwise interactions between the adsorbate atom and the substrate atoms, the coefficients show fast monotonous decay $^{20}$ and a few leading terms are enough for an accurate description of such systems. On the other hand, for more complex interaction potentials, the behavior of Fourier coefficients is not known and density-functional theory (DFT) calculations can be used to obtain them. ${ }^{24,38}$ In this study, we are interested in the response of an infinite rigid hexagonal monolayer to changes in $V(\mathbf{r})$. A hexagonal monolayer can be completely described by four variables: $R$-interadsorbate distance, $\alpha$-rotation angle, and $\left(x_{0}, y_{0}\right)$-the position on the substrate surface. The position of every atom in a nonrotated hexagonal monolayer is the linear combination of two vectors $l_{1}=R(2,0)$ and $l_{2}=R(1, \sqrt{3})$. If the rotation of the monolayer by angle $\alpha$ is allowed, the position of an atom becomes

$$
\begin{aligned}
\mathbf{r}= & {\left[x_{0}+R(2 a+b) \cos \alpha-\sqrt{3} R b \sin \alpha, y_{0}+\sqrt{3} R b \cos \alpha\right.} \\
& +R(2 a+b) \sin \alpha],
\end{aligned}
$$

where $a$ and $b$ are integers. Explicitly putting the dependence of $V(\mathbf{r})$ into Eq. (1) gives

$$
V\left(a, b, R, \alpha, x_{0}, y_{0}\right)=V_{0}+\sum_{G} V_{G} \exp \left(-{ }_{1} \mathbf{G r}\right) .
$$

Furthermore, it can be shown that $\exp (-1 \mathbf{G r})$ can be written in the following way:

$$
\begin{aligned}
\exp \left(-{ }_{1} \mathbf{G r}\right)= & \exp \left\{-{ }_{1}\left[\mathbf{G r}_{0}+C_{a}(R, \alpha, \mathbf{G}) a+C_{b}(R, \alpha, \mathbf{G}) b\right]\right\} \\
= & \exp \left(-{ }_{1} \mathbf{G r}_{0}\right) \times \exp \left[-{ }_{1} C_{a}(R, \alpha, \mathbf{G}) a\right] \\
& \times \exp \left[-{ }_{1} C_{b}(R, \alpha, \mathbf{G}) b\right]
\end{aligned}
$$

where $\mathbf{r}_{\mathbf{0}}=\left(x_{0}, y_{0}\right)$, while $C_{a}$ and $C_{b}$ are the terms associated with $a$ or $b$, respectively.

We would like to find a set of $\left(R, \alpha, x_{0}, y_{0}\right)$ for which

$$
E\left(R, \alpha, x_{0}, y_{0}\right)=\sum_{a=-\infty}^{\infty} \sum_{b=-\infty}^{\infty} V\left(a, b, R, \alpha, x_{0}, y_{0}\right)
$$

is minimized. In reality we are not interested in the energy of the infinite monolayer, as it has infinite value, but the quantity we would like to minimize is the energy per atom. This can be accomplished by rewriting Eq. (5) as an infinite limit of a partial sum and taking into account that $V(a, b, R$, $\left.\alpha, x_{0}, y_{0}\right)$ is separable into $V_{a}\left(a, R, \alpha, x_{0}, y_{0}\right)$ and $V_{b}(b, R, \alpha$, $\left.x_{0}, y_{0}\right)$, which depend on $a$ or $b$, respectively [see Eq. (4)]. By using these facts, two infinite sums in Eq. (5) can be converted into a product of two infinite limits of a partial sum,

$$
\begin{aligned}
E_{\text {atom }}\left(R, \alpha, x_{0}, y_{0}\right)= & \lim _{m \rightarrow \infty} \frac{1}{m^{2}} \sum_{k=0}^{m} \sum_{a=-k}^{k} V_{a}\left(a, R, \alpha, x_{0}, y_{0}\right) \\
& \times \lim _{m \rightarrow \infty} \frac{1}{m^{2}} \sum_{k=0}^{m} \sum_{b=-k}^{k} V_{b}\left(b, R, \alpha, x_{0}, y_{0}\right),
\end{aligned}
$$

where $m^{2}$ is the number of terms in the double sums, which comes from resolving a recursion equation.

In order to find the minimizers of Eq. (6), the following identity is used:

$$
\lim _{m \rightarrow \infty} \frac{1}{m^{2}} \sum_{k=0}^{m} \sum_{a=-k}^{k} \exp (-1 a x)=\delta(x-2 \pi n),
$$

where $\delta$ is the Dirac delta function. This identity can be proved by seeing that the exponential terms grow slower than $m^{2}$, unless $x=2 \pi n$, with integer $n$.

By using Eq. (7), only the terms where $C_{a}=2 \pi n$ and $C_{b}$ $=2 \pi k$ simultaneously are nonzero in Eq. (6). Therefore a system of two coupled equations must be solved for each Fourier term in Eq. (3), starting from $V_{G_{0}}$, with $G_{0}=\frac{4 \pi}{\sqrt{3} l}$, where $l$ is the substrate surface lattice constant. For example, the first Fourier term with length $G_{0}$ is $\cos \left(G_{0} y\right)$. Taking the $y$ component of real-space vector $\mathbf{r}$ from Eq. (2), the system of coupled equations becomes 

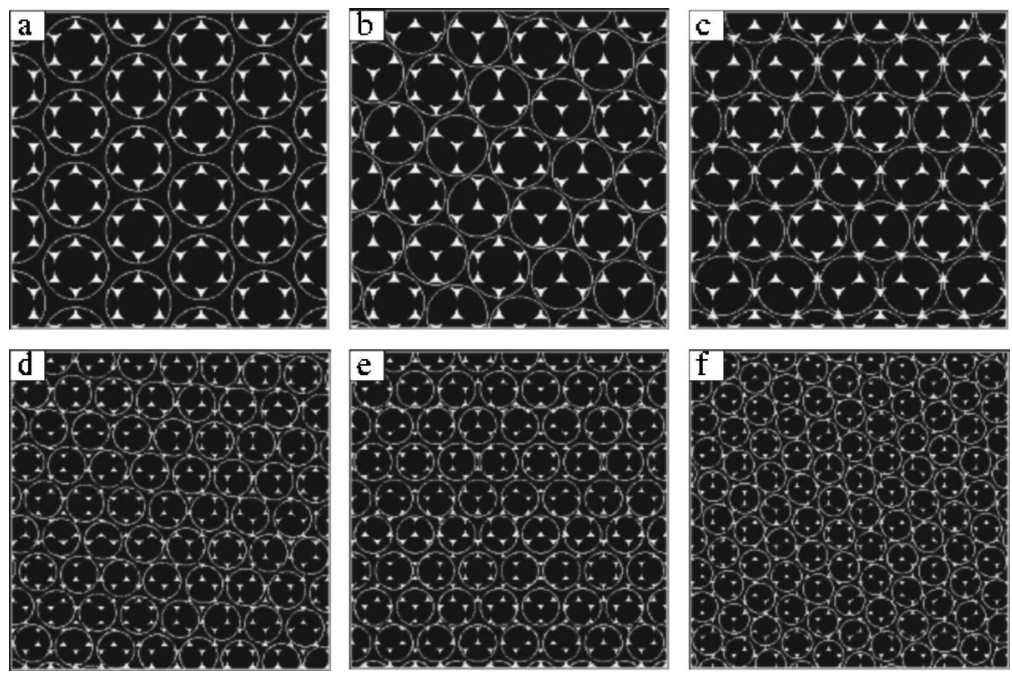

FIG. 1. Models of stable structures for Fourier terms with six shells of reciprocal cell vectors. Black filled circles are the substrate atoms, while larger white circles are the adsorbate atoms. The $\left(x_{0}, y_{0}\right)=(0,0)$ case is shown, which is obtained by putting one adsorbate atom directly on top of a substrate atom. The number of different structures with equal symmetry (same atomic registry but different interatomic distance) for $l \leq R$ $\leq 2 \sqrt{3} l$ is given in parentheses and they are explicitly specified in Table I. (a) Stable for the six shells (6). (b) Stable for the second and fifth shells (8). (c) Stable for the third and sixth shells (11). (d) Stable for fourth shell (42). (e) Stable for the fifth shell (22). (f) Stable for the sixth shell (22).

$$
\begin{gathered}
2 G_{0} R \sin \alpha=2 \pi n, \\
G_{0} R(\sqrt{3} \cos \alpha+\sin \alpha)=2 \pi k .
\end{gathered}
$$

This system has an infinite number of solutions, but in reality we are only interested in an interval of $R$. Typically, for atomic and small molecule adsorption, $l \leq R \leq 2 \sqrt{3} l$. This interval corresponds to the coverage range $\frac{1}{12} \leq \theta \leq 1$.

We have analyzed the solutions for the Fourier terms with six shells of the reciprocal cell vectors, starting with vectors of length $G_{0}$. When $R$ is restricted to a closed interval, $E_{\text {atom }}$ has a finite number of minima, due to the identity in Eq. (7). One could also notice that $R$ and $\alpha$ are decoupled from $\left(x_{0}, y_{0}\right)$, by observing the identity in Eq. (4). We have found that Fourier terms with reciprocal vectors of same length yield the same solutions. Therefore each shell of the reciprocal cell vectors gives a certain number of solutions. The first shell of vectors with length $G_{0}$ makes the simply commensurate structures with one atom per unit cell stable. For $l$ $\leq R \leq 2 \sqrt{3} l$, six $(R, \alpha)$ solution pairs exist: $(1,0),(\sqrt{3}, 30)$, $(2,0),(\sqrt{7}, 19.11),(3,0),(2 \sqrt{3}, 30)$. The $R$ values are in units of the substrate lattice constant and $\alpha$ is in degrees. The names of these structures in traditional surface science notation $^{1}$ are $(1 \times 1), \quad(\sqrt{3} \times \sqrt{3}) R 30^{\circ}, \quad(2 \times 2), \quad(\sqrt{7}$ $\times \sqrt{7}) R 19.11^{\circ},(3 \times 3)$, and $(2 \sqrt{3} \times 2 \sqrt{3}) R 30^{\circ}$, respectively. The stable $\left(x_{0}, y_{0}\right)$ position of the adsorbate layer depends on the sign of the $V_{G_{0}}$ coefficient: for $V_{G_{0}}>0$, the energy minimum is at threefold site, while for $V_{G_{0}}<0$ the minimum is located at on-top site.

Higher-order Fourier terms make the high-order commensurate (HOC) structures also stable. One interesting fact is that the simply commensurate structures, described above, are stable for every Fourier term. Therefore independently of the values of the $V_{G}$ coefficients, the simply commensurate structures will always be associated with global energy minima. The second shell of the reciprocal cell vectors makes commensurate structures with three atoms per unit cell stable. The third shell makes hexagonal structures with four atoms per unit cell stable. The structures with three atoms per unit cell are again minima for the fifth shell, while the structures with four atoms per unit cell become minima again until the sixth shell of the reciprocal cell vectors. The models for all minimum structures, which appear for any of the six shells, are shown in Fig. 1. Figure 2 shows same structures as Fig. 1 in a topographic way of presentation (see figure caption for more details). Table I shows $(R, \alpha)$ pairs for stable structures obtained for any of the Fourier terms.

The polar plot of all minimum $(R, \alpha)$ pairs is shown in Fig. 3. The size of the circle is provided just as a guide to the eye and indicates the relative energy of a certain minimum structure assuming a simple decaying behavior of Fourier coefficients (the larger the circle, the lower the energy). The circles are not drawn to scale and they just provide a qualitative energetic meaning. The simply commensurate structures are stable for all six shells of reciprocal vectors analyzed in this study, therefore they are indicated with the largest circle. The $V_{G}$ coefficients in any real system will clearly change the energy of the minimum structures. However, it is important to note that the minimum $\left(R, \alpha, x_{0}, y_{0}\right)$ values do not depend on the $V_{G}$ 's, the coefficients only change the relative stability of different structures. Figure 3 shows an interesting trend among the stable structures,
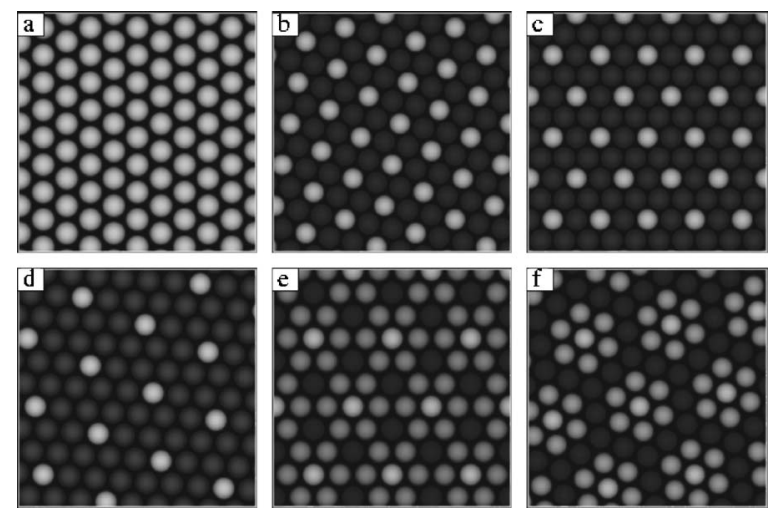

FIG. 2. Same structures as in Fig. 1 presented in topographic view. The color scale goes from bright (on-top site) to dark (threefold site). Note the decrease in symmetry with higher-order Fourier terms. The images were generated by ALSA software, see Ref. 39 for more details. 
TABLE I. $(R, \alpha)$ values for stable structures for any combination of Fourier terms, up to the sixth shell of reciprocal cell vectors (RCV) for $l \leq R \leq 2 \sqrt{3} l$, where $l$ is the substrate surface lattice constant. $R$ is reported in units of $l$, while $\alpha$ is reported in degrees. The first row indicates the simple commensurate structures with one atom per unit cell, which are stable for any of the sixth shells of RCV. The second row shows the structures with three atoms per unit cell. The stable structures for other high-order Fourier terms follow.

\begin{tabular}{|c|c|c|c|c|c|}
\hline \multirow[b]{2}{*}{ All Six } & \multicolumn{5}{|c|}{$(R, \alpha)$} \\
\hline & $\begin{array}{c}(1,0.00) \\
(2 \sqrt{3}, 30.00)\end{array}$ & $(\sqrt{3}, 30.00)$ & $(2,0.00)$ & $(\sqrt{7}, 19.11)$ & $(3,0.00)$ \\
\hline \multirow[t]{2}{*}{$2 \mathrm{nd}+5 \mathrm{th}$} & $(2 / \sqrt{3}, 30.00)$ & $(1.5275,10.89)$ & $(2.0817,16.10)$ & $(2.3094,30.00)$ & $(2.5166,6.59)$ \\
\hline & $(2.8868,30.00)$ & $(3.0551,10.89)$ & $(3.2146,21.05)$ & & \\
\hline \multirow[t]{3}{*}{$3 \mathrm{rd}+6$ th } & $(1.3229,19.11)$ & $(1.5000,0.00)$ & $(1.8028,13.90)$ & $(2.1794,23.41)$ & $(2.2913,10.89)$ \\
\hline & $(2.5000,0.00)$ & $(2.5981,30.00)$ & $(2.7839,8.95)$ & $(3.0414,25.28)$ & $(3.1225,16.10)$ \\
\hline & $(3.2787,7.59)$ & & & & \\
\hline \multirow[t]{9}{*}{ 4th } & $(1.0000,21.79)$ & $(1.1339,19.11)$ & $(1.3093,10.89)$ & $(1.3628,27.00)$ & $(1.3628,5.21)$ \\
\hline & $(1.5119,19.11)$ & $(1.6475,17.48)$ & $(1.6475,4.31)$ & $(1.7321,8.21)$ & $(1.8898,19.11)$ \\
\hline & $(1.9640,10.89)$ & $(2.0000,21.79)$ & $(2.1044,10.16)$ & $(2.1044,28.05)$ & $(2.2678,19.11)$ \\
\hline & $(2.2991,15.61)$ & $(2.2991,6.18)$ & $(2.3604,24.79)$ & $(2.3604,3.00)$ & $(2.4785,11.52)$ \\
\hline & $(2.4785,26.70)$ & $(2.6186,10.89)$ & $(2.6458,2.68)$ & $(2.7255,27.00)$ & $(2.7255,5.21)$ \\
\hline & $(2.8536,12.52)$ & $(2.8536,25.69)$ & $(2.9520,14.56)$ & $(2.9520,7.22)$ & $(3.0000,21.79)$ \\
\hline & $(3.0237,19.11)$ & $(3.0938,28.68)$ & $(3.0938,6.89)$ & $(3.2293,13.29)$ & $(3.2293,24.92)$ \\
\hline & $(3.2733,10.89)$ & $(3.2950,17.48)$ & $(3.2950,4.31)$ & $(3.3594,2.11)$ & $(3.3594,23.90)$ \\
\hline & $(3.4017,19.11)$ & $(3.4641,8.21)$ & & & \\
\hline \multirow[t]{5}{*}{5 th } & $(1.2019,13.90)$ & $(1.3333,0.00)$ & $(1.4530,23.41)$ & $(1.6667,0.00)$ & $(1.7638,19.11)$ \\
\hline & $(1.8559,8.95)$ & $(2.0276,25.28)$ & $(2.1858,7.59)$ & $(2.3333,0.00)$ & $(2.3333,21.79)$ \\
\hline & $(2.4037,13.90)$ & $(2.6034,26.33)$ & $(2.6667,0.00)$ & $(2.7285,12.22)$ & $(2.8480,5.82)$ \\
\hline & $(2.9059,23.41)$ & $(2.9627,17.00)$ & $(3.1798,27.00)$ & $(3.1798,5.21)$ & $(3.2830,15.30)$ \\
\hline & $(3.3333,0.00)$ & $(3.3830,9.83)$ & & & \\
\hline \multirow[t]{5}{*}{ 6th } & $(1.0408,16.10)$ & $(1.2583,6.59)$ & $(1.4434,30.00)$ & $(1.6073,21.05)$ & $(1.7559,4.72)$ \\
\hline & $(1.8930,22.41)$ & $(2.0207,30.00)$ & $(2.0207,8.21)$ & $(2.2546,3.67)$ & $(2.3629,17.78)$ \\
\hline & $(2.4664,24.18)$ & $(2.5658,13.00)$ & $(2.7538,24.79)$ & $(2.7538,3.00)$ & $(2.8431,14.70)$ \\
\hline & $(2.9297,20.17)$ & $(3.0139,5.50)$ & $(3.1754,30.00)$ & $(3.2532,2.54)$ & $(3.3292,12.52)$ \\
\hline & $(3.3292,25.69)$ & $(3.4034,17.27)$ & & & \\
\hline
\end{tabular}

namely hexagonal pattern can be clearly identified if points corresponding to minimum structures from the same shell of reciprocal vectors are connected.

It is also worth noticing that the lower-order Fourier terms vanish for HOC structures with a certain number of atoms per unit cell. For example, the first Fourier term vanishes for all rigid hexagonal structures with more than one atom per unit cell. The first and second Fourier terms vanish for a structure with more than three atoms per unit cell. This conclusion can be further extended by observing the HOC structures in Figs. 1 and 2 and their relation to specific Fourier terms.

\section{COMPARISON WITH REAL EXPERIMENTAL SYSTEMS}

In order to verify if our model could describe any real experimental results, we made comparison with iodine adsorbed on the Pt(111) surface and $\mathrm{K}, \mathrm{Rb}$, and $\mathrm{Cs}$ adsorbed on the $\mathrm{Ag}(111)$ surface. Iodine on the $\mathrm{Pt}(111)$ surface is known to form four different commensurate structures: $(\sqrt{3}$ $\times \sqrt{3}) R 30^{\circ}$ with one atom per unit cell, $(\sqrt{7} \times \sqrt{7}) R 19.11^{\circ}$ with three atoms per unit cell, $(3 \times 3)-$ sym and $(3 \times 3)$ - asym with four atoms per unit cell. ${ }^{16,40,41}$ The alkalis form wide variety of commensurate and incommensurate structures on the $\mathrm{Ag}(111)$ surface. ${ }^{14,42}$ Both systems possess several different high-order commensurate structures, which indicates the complexity of the Fourier expansion of the adsorbate-substrate interaction potential. Interestingly, the adsorbate-adsorbate interaction is thought to be dominated by repulsive forces in both cases. For alkali atoms, the charge-transfer effects along with the substrate-mediated interactions are presumably responsible for an effective repulsive interaction, ${ }^{14,42,43}$ while several forces contribute in the case of interhalogen interactions. ${ }^{16,27,44,45}$ As it was already mentioned above, the repulsive forces favor the formation of hexagonal monolayers. Therefore the theory developed here would seem to be most suitable for such kind of system. Figure 4 shows the same data of Fig. 3 along with several possible trajectories ${ }^{53}$ for $\mathrm{I}-\mathrm{Pt}(111)$ and $\mathrm{K}, \mathrm{Rb}$, and $\mathrm{Cs}$ on $\mathrm{Ag}(111)$. Clearly, the experimentally observed trajectories 


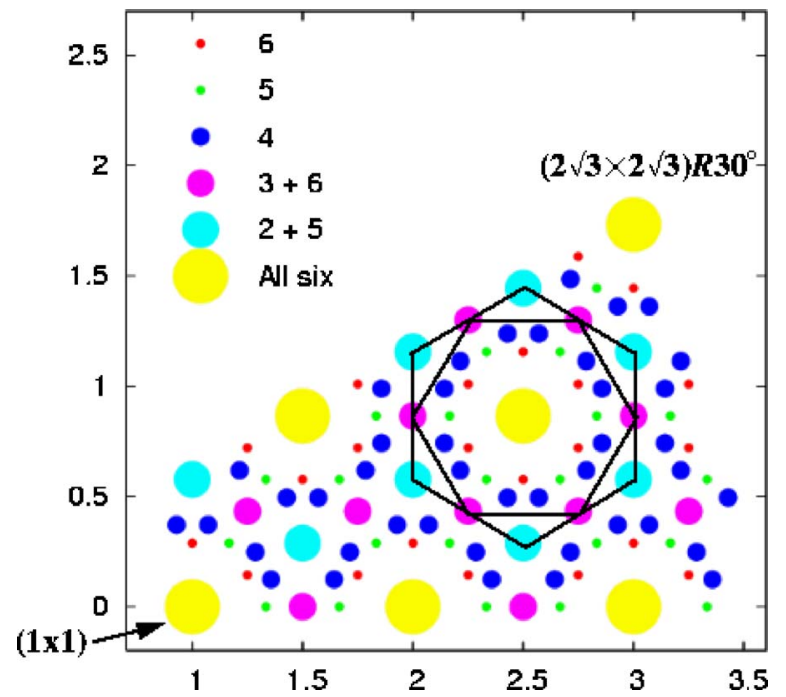

FIG. 3. (Color online) $(R, \alpha)$ plot of stable structures in polar coordinates. The values of variables are $l \leq R \leq 2 \sqrt{3} l$ and $0^{\circ} \leq \alpha$ $\leq 30^{\circ}$. This limit can be imposed on $\alpha$ due to the hexagonal symmetry of the monolayer. The figure legend specifies the reciprocal vector shells for which a certain structure is a minimum. Hexagonal patterns can be clearly identified if the points which belong to minimum structures with the same symmetry are connected. The size of the circle is provided just as a guide to the eye, assuming that the Fourier coefficients show decaying behavior. Namely, the size changes from 6 to 1 .

depend on the exact values of the $V_{G}$ coefficients and the adsorbate-adsorbate interactions. Therefore the results presented here can be regarded at most qualitative. However, several interesting points can be made as a result of comparison of this analytic model with real experimental systems. It

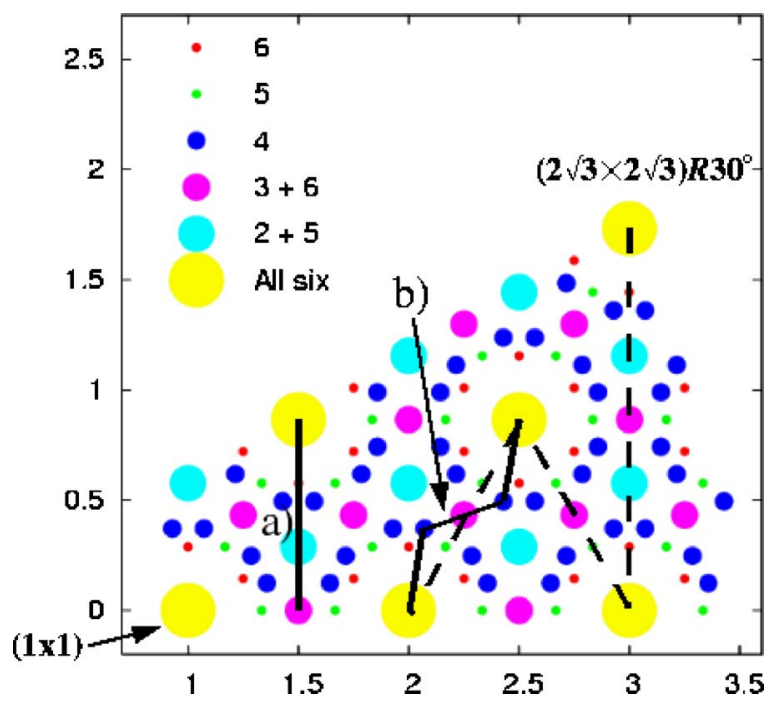

FIG. 4. (Color online) Same information as in Fig. 3 with several proposed trajectories for $\mathrm{I}-\mathrm{Pt}(111)$ and $\mathrm{K}, \mathrm{Rb}$, and $\mathrm{Cs}$ on $\operatorname{Ag}(111)$. One possible trajectory for I-Pt(111) is marked by a). The dashed line indicates a simple linear trajectory of rotational epitaxy for alkalis on the $\operatorname{Ag}(111)$ surface. Close agreement with experimental data of Leatherman et al. (Ref. 14) can be obtained by following the trajectory $b$ ). is worth to observe that in the case of I-Pt(111), the obvious trajectory is a straight line in polar coordinates of the corresponding $(R, \alpha)$ space. For $\mathrm{K}, \mathrm{Rb}$, and $\mathrm{Cs}$ on $\operatorname{Ag}(111)$, several trajectories are possible between $(2 \times 2)$ and $(\sqrt{7}$ $\times \sqrt{7}) R 19.11^{\circ}$, passing through different high-order commensurate (HOC) structures. Close agreement to experimental results of Leatherman et al. ${ }^{14}$ can be obtained by following the trajectory marked by (b) in Fig. 4, which transformed into Cartesian coordinates in $(R, \alpha)$ space, gives the experimental $\alpha$ vs $R$ curve. However, in order to prove that adsorbate-substrate interactions play a decisive role in determining the rotational epitaxy of alkalis on $\operatorname{Ag}(111)$, the exact values of $V_{G}$ coefficients should be calculated for $(2 \times 2)$ and $(\sqrt{7} \times \sqrt{7}) R 19.11^{\circ}$ structures. This should be possible by employing density-functional theory (DFT) calculations, which have been frequently used for halogens ${ }^{24,27,44,46-48}$ and alkalis $^{43,49,50}$ adsorbed on metal surfaces.

Doering ${ }^{9}$ proposed a model based on symmetry considerations of the monolayer and identified a special trajectory in the $(R, \alpha)$ space for hexagonal monolayer on hexagonal substrate. Grey and Bohr ${ }^{10,11}$ used a one-component Fourier expansion and, assuming a finite-size effect due to surface terraces, found a similar trajectory proposed by Doering. One should note that the theory developed in this paper extends the models by Doering and Grey-Bohr by taking several shells of the reciprocal cell vectors into account. Clearly, the $V_{G}$ coefficients introduce additional degrees of freedom for the possible trajectories in the $(R, \alpha)$ space.

\section{DISCUSSION}

Before going into further discussion, it should be emphasized that at the present time no single model can describe the rich epitaxial behavior of adsorbed monolayers. Hereby the effect of adsorbate-substrate interaction on the hexagonal monolayer adsorbed on hexagonal-substrate is analyzed, which was generally overlooked by previous theories. We show that a rigorous analytical treatment is possible when the adsorbate-substrate interaction is described by Fourier expansion with any number of terms. It still remains to be seen if the adsorbate-adsorbate interaction can be included analytically or a numerical procedure should be developed for this purpose. However, it should be mentioned that analytic expressions for adsorbate-adsorbate interactions are only known for a few physisorption systems. ${ }^{31,51,52}$ In the case of chemisorption, i.e., for halogens and alkalis adsorbed on metal surfaces, even a qualitative picture of interactions is not available. Although, in its present form, the theory lacks consideration of interactions between the adsorbate atoms, it should be exact when the interaction around commensurate structures is repulsive and strong enough to form rigid hexagonal monolayers.

Figure 4 identifies several possible trajectories in the $(R, \alpha)$ space of hexagonal monolayers, which connect the experimentally observed structures. These linear trajectories correspond to models of Doering ${ }^{9}$ and Grey-Bohr, ${ }^{10,11}$ which used symmetry considerations and finite-size effects respectively, to explain the rotation behavior of hexagonal mono- 
layers. We show that for alkalis on $\operatorname{Ag}(111)$ different trajectory is required to fit experimental results. In general, many pathways are possible depending on the values of the Fourier coefficients. Detailed description of these pathways could be important for understanding of phase transitions in adsorbed monolayers.

Several microscopic theories have been proposed for detailed characterization of structures of adsorbed monolayers and phase transitions between them. One of the most studied systems are the square monolayers on square and rectangular substrates. ${ }^{2-4}$ However, the adsorbate-substrate interaction potential has been generally described as a one-parameter Fourier expansion or in terms of the Lennard-Jones potential. The interaction between the adsorbate species has been assumed to be of Lennard-Jones type as well. It would be interesting to see how the results of these studies are affected if larger Fourier expansion is used for the description of the adsorbate-substrate interaction potential.

\section{CONCLUSIONS}

An analytic theory of hexagonal monolayer interacting with hexagonal substrate is presented. The interaction is described by a Fourier expansion with up to six shells of reciprocal cell vectors. Several simple commensurate and highorder commensurate (HOC) structures are found to be stable, and a clear correspondence between energy and symmetry of the adsorbed monolayer is established. Preliminary compari- son with experimental epitaxial behavior of real systems, such as iodine adsorbed on $\mathrm{Pt}(111)$ surface and alkalis on $\mathrm{Ag}(111)$ surface is promising. Several trajectories are identified in $(R, \alpha)$ space of the hexagonal monolayers, which seem to describe the experimental data rather well. However, one should keep in mind that coefficients of the Fourier expansion must be calculated in order to make better comparison. DFT calculations should be most adequate for such purpose and could be readily performed. The present study identifies adsorbate-substrate interaction as an important contribution for understanding detailed behavior of monolayer epitaxy on well-ordered surfaces. Furthermore, I believe that this theory is exact when the interaction between adsorbate species is repulsive around commensurate structures and strong enough to form rigid hexagonal monolayers. Extensions of this theory to different monolayer or substrate symmetry are straightforward.

\section{ACKNOWLEDGMENTS}

The author acknowledges Laura Prat and Hanne Schultz for discussions about some mathematical aspects of this paper, Nikola Batina for revision of the initial draft, and the organizers of the program Bridging Time and Length Scales in Materials Science and Bio-Physics which was held at the Institute for Pure and Applied Mathematics at the University of California Los Angeles. Financial support was provided by CONACYT, IPAM/UCLA, and LUFAC Computación.
*Electronic address: sanix @ixil.izt.uam.mx

${ }^{1}$ G. A. Somorjai, Introduction to Surface Chemistry and Catalysis (Wiley, New York, 1994).

${ }^{2}$ O. Biham, L.-W. Chen, and G. Vidali, Surf. Sci. 287, 815 (1993).

${ }^{3}$ A. Patrykiejew, S. Sokolowski, and K. Binder, Surf. Sci. Rep. 37, 207 (2000).

${ }^{4}$ A. Patrykiejew, S. Sokolowski, and K. Binder, J. Chem. Phys. 115, 983 (2001).

${ }^{5}$ A. C. Hillier and M. D. Ward, Phys. Rev. B 54, 14037 (1996).

${ }^{6}$ D. E. Hooks, T. Fritz, and M. D. Ward, Adv. Mater. (Weinheim, Ger.) 13, 227 (2001).

${ }^{7}$ A. D. Novaco and J. P. McTague, Phys. Rev. Lett. 38, 1286 (1977).

${ }^{8}$ J. P. McTague and A. D. Novaco, Phys. Rev. B 19, 5299 (1979).

${ }^{9}$ D. L. Doering, J. Vac. Sci. Technol. A 3, 809 (1985).

${ }^{10}$ F. Grey and J. Bohr, Europhys. Lett. 18, 717 (1992).

${ }^{11}$ F. Grey and J. Bohr, in Phase Transitions in Surface Films 2, edited by H. Taub, G. Torzo, H. J. Lauter, and S. C. Fain (Plenum Press, New York, 1991), p. 83.

${ }^{12}$ H. Shiba, J. Phys. Soc. Jpn. 46, 1852 (1979).

${ }^{13}$ H. Shiba, J. Phys. Soc. Jpn. 48, 211 (1980).

${ }^{14}$ G. S. Leatherman and R. D. Diehl, Phys. Rev. B 53, 4939 (1996).

${ }^{15}$ G. S. Leatherman, M. Karimi, G. Vidali, and R. D. Diehl, Phys. Rev. B 56, 6970 (1997).

${ }^{16}$ O. M. Magnussen, Chem. Rev. (Washington, D.C.) 102, 679 (2002).

${ }^{17}$ C. A. Lucas, N. M. Marković, and P. N. Ross, Phys. Rev. B 55,
7964 (1997).

${ }^{18}$ B. M. Ocko, G. M. Watson, and J. Wang, J. Phys. Chem. 98, 897 (1994).

${ }^{19}$ O. M. Magnussen, B. M. Ocko, R. R. Adzic, and J. X. Wang, Phys. Rev. B 51, 5510 (1995).

${ }^{20}$ W. A. Steele, Surf. Sci. 36, 317 (1973).

${ }^{21}$ J. M. Gottlieb and L. W. Bruch, Phys. Rev. B 44, 5750 (1991).

${ }^{22}$ J. M. Gottlieb, Phys. Rev. B 42, 5377 (1990).

${ }^{23}$ G. Vidali and M. W. Cole, Phys. Rev. B 29, 6736 (1984).

${ }^{24}$ A. Tkatchenko (unpublished).

${ }^{25}$ L. W. Bruch, M. W. Cole, and E. Zaremba, Physical Adsorption: Forces and Phenomena (Oxford University Press, New York, 1997).

${ }^{26}$ K. Kern, Phys. Rev. B 35, 8265 (1987).

${ }^{27}$ A. Tkatchenko, N. Batina, A. Cedillo, and M. Galvan, Surf. Sci. 581, 58 (2005).

${ }^{28}$ A. Tkatchenko, N. Batina, and M. Galvan, Phys. Rev. Lett. 97, 036102 (2006).

${ }^{29}$ J. L. F. D. Silva, C. Stampfl, and M. Scheffler, Phys. Rev. Lett. 90, 066104 (2003).

${ }^{30}$ J. L. F. D. Silva, C. Stampfl, and M. Scheffler, Phys. Rev. B 72, 075424 (2005).

${ }^{31}$ L. W. Bruch, A. P. Graham, and J. P. Toennies, J. Chem. Phys. 112, 3314 (1999).

${ }^{32}$ A. K. Dham, W. J. Meath, A. R. Allnatt, R. A. Aziz, and M. J. Slaman, Chem. Phys. 142, 173 (1990).

${ }^{33}$ J. F. Zhu, H. Ellmer, H. Malissa, T. Brandstetter, D. Semrad, and 
P. Zeppenfeld, Phys. Rev. B 68, 045406 (2003).

${ }^{34}$ B. Vogt, B. Kessler, N. Müller, G. Schönhense, B. Schmiedeskamp, and U. Heinzmann, Phys. Rev. Lett. 67, 1318 (1991).

${ }^{35}$ G. Hilgers, M. Pothoff, N. Müller, and U. Heinzmann, Surf. Sci. 322, 207 (1995).

${ }^{36}$ R. D. Diehl, T. Seyller, M. Caragiu, G. S. Leatherman, N. Ferralis, K. Pussi, P. Kaukasoina, and M. Lindroos, J. Phys.: Condens. Matter 16, 2839 (2004).

${ }^{37}$ A. D. McLachlan, Mol. Phys. 7, 381 (1964).

${ }^{38}$ N. Takeuchi, C. T. Chan, and K. M. Ho, Phys. Rev. Lett. 63, 1273 (1989).

${ }^{39}$ A. Tkatchenko and N. Batina, J. Chem. Phys. 122, 094705 (2005).

${ }^{40}$ B. C. Schardt, S. L. Yau, and F. Rinaldi, Science 243, 1050 (1989).

${ }^{41}$ S. L. Yau, C. M. Vitus, and B. C. Schardt, J. Am. Chem. Soc. 112, 3677 (1990).

${ }^{42}$ R. D. Diehl and R. McGrath, J. Phys.: Condens. Matter 9, 951
(1997).

${ }^{43}$ J. Neugebauer and M. Scheffler, Phys. Rev. B 46, 16067 (1992).

${ }^{44}$ S. A. Wasileski and M. J. Weaver, J. Phys. Chem. B 106, 4782 (2002).

${ }^{45}$ J. Lipkowski, Z. Shi, A. Chen, B. Pettinger, and C. Bilger, Electrochim. Acta 43, 2875 (1998).

${ }^{46}$ M. T. M. Koper and R. A. van Santen, Surf. Sci. 422, 118 (1999).

${ }^{47}$ S. Wang and P. A. Rikvold, Phys. Rev. B 65, 155406 (2002).

${ }^{48}$ S. J. Mitchell and M. T. M. Koper, Surf. Sci. 563, 169 (2004).

${ }^{49}$ C. Stampfl and M. Scheffler, Surf. Rev. Lett. 2, 317 (1995).

${ }^{50}$ A. Kiejna, T. Ossowski, and E. Wachowicz, Surf. Sci. 548, 22 (2004).

${ }^{51}$ J. A. Barker and C. T. Rettner, J. Chem. Phys. 97, 5844 (1992).

${ }^{52}$ J. A. Barker and C. T. Rettner, J. Chem. Phys. 101, 9202 (1994).

${ }^{53}$ The word trajectory is used to indicate the rotation angle vs interatomic distance function, which connects any given two structures and could pass through other HOC structures on its way. 